\title{
Incorporation of SemiSpan SuperSonic Transport (S4T) Aeroservoelastic Models into SAREC-ASV Simulation
}

\author{
David M. Christhilf ${ }^{1}$ \\ Lockheed-Martin, Langley Program Office, Hampton, VA, 23681-2199 \\ and \\ Anthony S. Pototzky ${ }^{2}$ and William L. Stevens ${ }^{3}$ \\ NASA-Langley Research Center, Hampton, VA, 23681-2199
}

\begin{abstract}
The Simulink-based Simulation Architecture for Evaluating Controls for Aerospace Vehicles (SAREC-ASV) was modified to incorporate linear models representing aeroservoelastic characteristics of the SemiSpan SuperSonic Transport (S4T) wind-tunnel model. The S4T planform is for a Technology Concept Aircraft (TCA) design from the 1990s. The model has three control surfaces and is instrumented with accelerometers and strain gauges. Control laws developed for wind-tunnel testing for Ride Quality Enhancement, Gust Load Alleviation, and Flutter Suppression System functions were implemented in the simulation. The simulation models open- and closed-loop response to turbulence and to control excitation. It provides time histories for closed-loop stable conditions above the open-loop flutter boundary. The simulation is useful for assessing the potential impact of closed-loop control rate and position saturation. It also provides a means to assess fidelity of system identification procedures by providing time histories for a known plant model, with and without unmeasured turbulence as a disturbance. Sets of linear models representing different Mach number and dynamic pressure conditions were implemented as MATLAB Linear Time Invariant (LTI) objects. Configuration changes were implemented by selecting which LTI object to use in a Simulink template block. A limited comparison of simulation versus wind-tunnel results is shown.
\end{abstract}

\section{Nomenclature}

$M \quad=\quad$ Mach Number, non-dimensional

$\bar{q} \quad=$ Dynamic Pressure, psf

\section{Introduction}

$\mathrm{T}$ HE Simulink-based Simulation Architecture for Evaluating Controls for Aerospace Vehicles (SAREC-ASV) ${ }^{1}$ has evolved over time as a means for organizing the components of an aircraft simulation in a hierarchical fashion that limits the complexity of any given component, and arranges components such that navigation from one to another is systematic. It has been used to host various aircraft simulations, and traces its start to a High Speed Civil Transport (HSCT) simulation developed in the mid $1990 \mathrm{~s}^{2}$. Once it was deemed that SAREC-ASV (or simply SAREC) had reached a useful level of maturity, it was used to host a textbook simulation of an F-16 ${ }^{3}$, chosen so that the model could be distributed without restriction. That model as implemented is a somewhat simplified version of a simulation developed for a real-time piloted simulation study in the late $1970 \mathrm{~s}^{4}$ and it contains six degree-offreedom equations of motion with tabular representations for aerodynamic and propulsion forces and moments. Feedback control laws and a MATLAB animation for aircraft position and orientation were included in the simulation.

\footnotetext{
${ }^{1}$ Research Engineer Staff, Langley Program Office, c/o NASA-LaRC, Mail Stop 308, Senior Member AIAA.

${ }^{2}$ Research Aerospace Engineer, Aeroelasticity Branch, Mail Stop 340, Senior Member AIAA.

${ }^{3}$ Aerospace Engineer, Aeroelasticity Branch, Mail Stop 340, Member AIAA.
}

1

American Institute of Aeronautics and Astronautics 
The current paper describes the adaptation of SAREC in order to host a simulation of the SemiSpan SuperSonic Transport (S4T) ${ }^{5}$ aeroelastically scaled wind-tunnel model (Fig. 1). The S4T was one of several models constructed in the 1990s as part of the High Speed Research (HSR) program, but it was not tested at that time due to termination of the program. It represents the Technology Concept Aircraft (TCA) configuration, and is representative of a class of vehicles with long, slender fuselage and thin, swept wing. The interaction of transonic shock effects, wing and fuselage flexibility, and the anisotropic properties of composite materials lead to challenges in characterizing the vehicle dynamic response. At the same time, active controls may be useful for this class of vehicle in order to provide flutter margins for the relatively brief transition through the transonic regime to supersonic cruise with less weight penalty than other alternatives. The S4T was eventually tested three times in the NASA Langley Research Center Transonic Dynamics Tunnel (TDT) ${ }^{5}$, from 2007 to 2009, as part of the Supersonics Project within the NASA Fundamental Aeronautics Program (FAP). The third entry included open and closed-loop Controller Performance Evaluation (CPE) ${ }^{6}$ and closed-loop testing of control laws for Ride Quality Enhancement (RQE), Gust Load Alleviation (GLA), and Flutter Suppression System (FSS) functions.

The SAREC S4T simulation was developed to support control-law integration and wind-tunnel testing. Since the S4T is wall mounted, its rigid body motion is constrained. Rather than six degrees-of-freedom, the S4T has only limited pitch and plunge freedom, restricted by the (approximately $1 \mathrm{~cm}$ ) range of travel of two vertical spring mounts and by a mechanism that restrains roll, yaw, lateral, and axial motion. The equations of motion for the S4T were implemented as linear state-space models with control deflection and turbulence inputs, accelerometer and cutting-plane load sensor outputs, and state variables representing the complex modes of vibration. ${ }^{\S}$ The simulation was used for generating time histories for open and closed-loop response to turbulence and response to control surface excitation, in the presence of actuator rate and position limit non-linearities. Turbulence models were designed to be representative of the TDT wind-tunnel environment. ${ }^{7}$

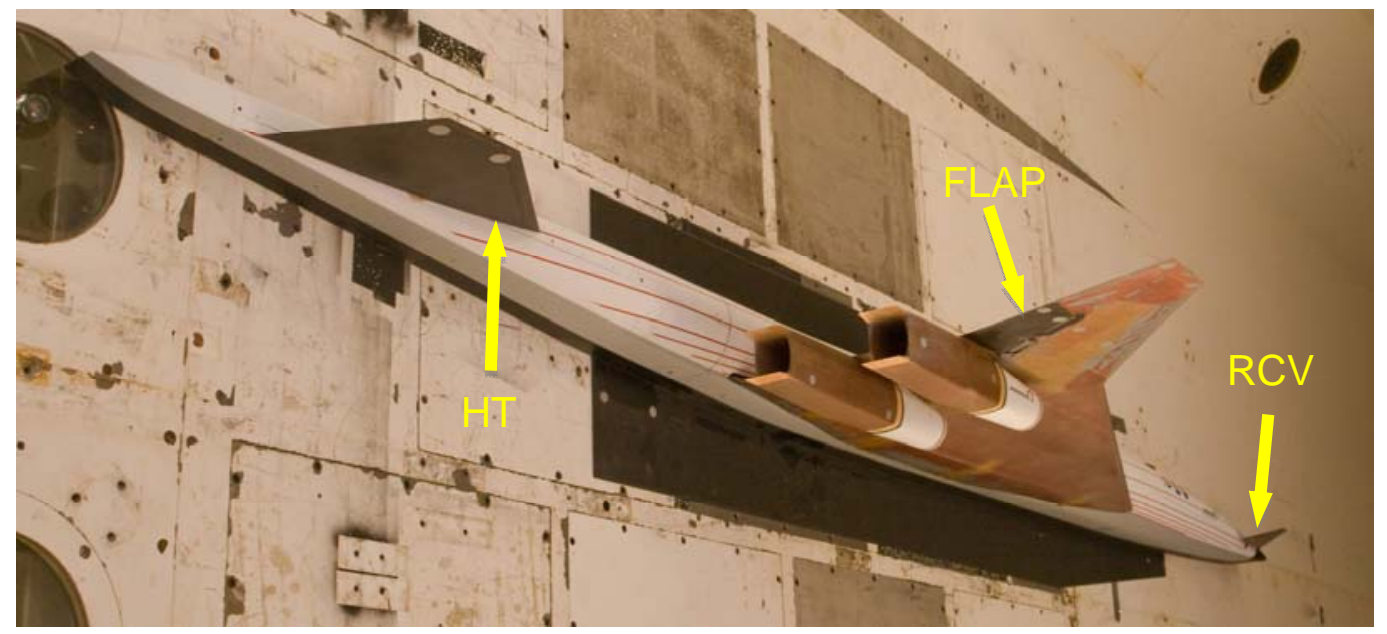

Figure 1. S4T In Wind-Tunnel. Aft view of wing, engine nacelles, aerodynamic fairing, allmoving horizontal tail (HT), flaperon (FLAP), ride control vane (RCV), and targets for videogrammetric model deformation (VMD) measurement system.

A key role for the simulation was to provide a means for verifying that control-law sign conventions, units and channel assignments were consistent with the actual wind-tunnel model. Additionally, the simulation provided an indication of whether actuator rate and position limits would be exceeded, closed-loop, in the presence of turbulence. It provided time histories from a known plant model, with and without turbulence present, that could be used to assess the fidelity of plant estimation tools that were also applied to actual wind-tunnel data. ${ }^{6}$ The simulation provided the means to generate closed-loop time histories for conditions that were open-loop unstable, for use in evaluating the estimation tools for conditions that are not currently available experimentally.

\footnotetext{
$\S$ The vibration modes that result from a structural analysis in the absence of air (in vacuo) have real-valued eigenvectors with stationary node lines. In the presence of an air flow the eigenvectors become complex, with node lines that can oscillate spatially within a finite range. If a mode changes such that the node lines actually travel downstream, as with a flag in the breeze, then there exists a mechanism that can extract energy from the air flow and become unstable.
} 
The plant models incorporated into the simulation were received as linear state space models, provided from three sources. NASA provided one set of state space models, and each of two companies contracted to provide control laws also provided state space plant models. The state space models were incorporated into MATLAB Linear Time Invariant (LTI) objects, which were implemented in the simulation by means of Simulink Configurable Subsystem blocks used as templates for the various LTI objects. Control laws provided by contract were required to be of a specified state space format. That format was processed into a standardized form that was used during windtunnel testing. The simulation made use of the identical form for the control laws, which could be loaded interchangeably. A simplified Graphical User Interface (GUI) was developed for the S4T Simulation to facilitate changing plant models and control laws, opening or closing the feedback loop, selecting the type of turbulence model or control surface excitation, running the simulation, and saving the results. Changes made to the simulation were tracked using the Subversion (SVN) ${ }^{8}$ software management tool.

Contracted development of the control laws and results of the control-law testing are reported on in Refs. 9, 10, and 11 but will not be discussed here. Methods for generating linear plant models, including treatment of control surface inputs, rigid body modes generally, and loads outputs, are reported in Refs. 12 and 13. Linear plant models are central to characterizing the aeroseroelastic response for the S4T, and characteristics of the models that have implications for incorporating into simulation are discussed in this paper. A brief comparison of simulation and wind-tunnel results is also presented.

\section{SemiSpan SuperSonic Transport}

The wing for the S4T is based on the TCA planform, with cranked leading-edge and trailing-edge sweep (Fig. 2). It is made of composite materials and is aeroelastically scaled to match the characteristics of the TCA wing. Two starboard engine nacelles are mounted on separate pylons beneath the wing, inboard of the trailing-edge crank, with non-functioning flow through engines. The S4T has three hydraulically driven control surfaces. An all-moving horizontal tail (HT) mounted on the aft fuselage was sized for low speed TCA pitch control, with control authority increasing at higher airspeeds. A smaller horizontal ride control vane (RCV) located near the pilot station was added to the TCA design for vibration suppression, through active controls, for improving flying qualities. The function of the RCV suggests that it was designed to be feathered so as to not carry a trim load, and therefore is not expected to generate substantial downwash on or vortex interaction with the wing. The third S4T control surface is a single flaperon (FLAP) located just outboard of the crank in the wing trailing-edge. The FLAP was designed for both symmetric deflection as a flap for high lift, and for antisymmetric deflection as an aileron for roll control. The wall mount boundary condition restrains antisymmetric structural modes, so the FLAP deflections are notionally symmetric for left and right sides, interacting with the symmetric aeroelastic modes. However, actuation bandwidths are more representative of an aileron than a typically slower flap. The TCA has an additional trailing-edge surface between the nacelles, and one inboard of the nacelles, that were not included in the S4T, either actively or statically, due in part to geometric and stiffness restrictions for the model scale wing. Of the three control surfaces that were

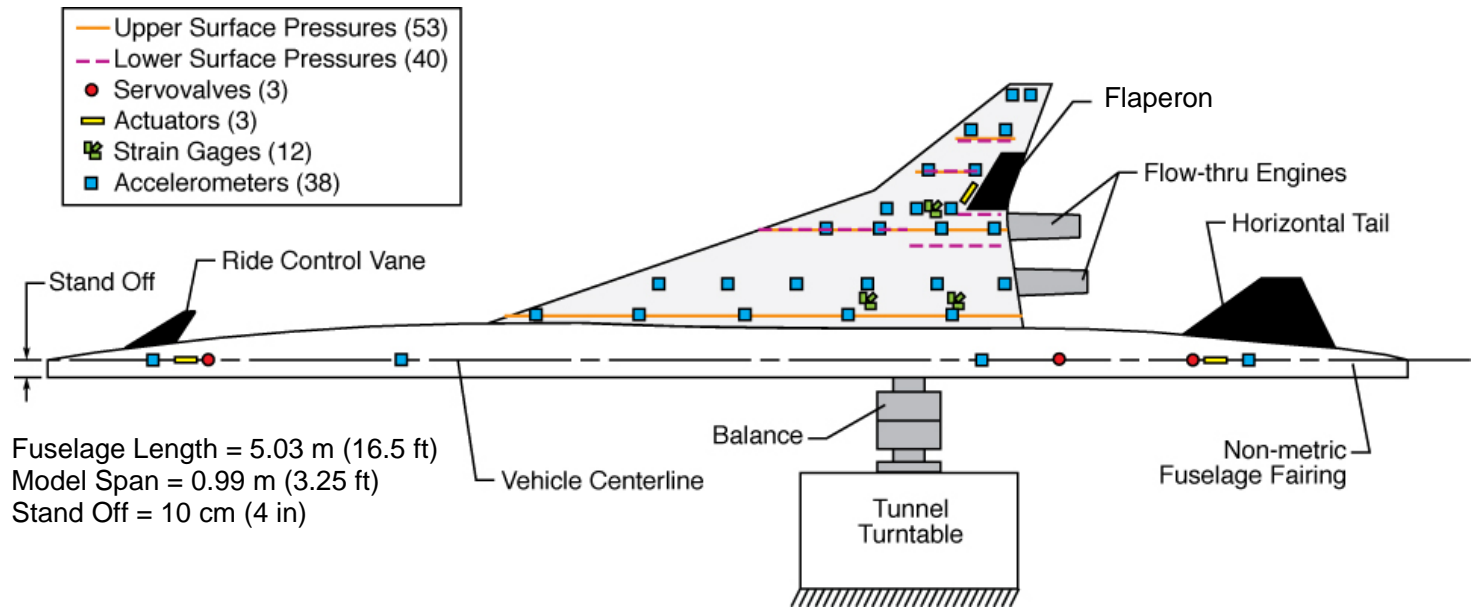

Figure 2. Instrumentation Layout. General depiction of mount system, wing, engine nacelles, fuselaae fairina, control surfaces, accelerometers, and strain aauaes. 
implemented, the RCV is the only one designed specifically for active aeroelastic control. The HT has the most control authority, and the FLAP surface area is only about 3.5\% of the exposed wing area, somewhat limiting its effectiveness.

Fuselage flexibility is represented by a tailored composite beam mounted to a rigid beam by means of two U-springs strategically placed to minimize the interference with vibration modes, and by two mechanisms for restraining roll and yaw rotations, and lateral and axial translations. The rigid beam in turn is supported by a load balance mounted to a turntable for adjusting angle of attack. The flexible and rigid beams are surrounded by a rigid aerodynamic fairing mounted such that it does not contribute loads to the load balance. Support shafts for the RCV and HT pass through holes in the fairing and are attached to the flexible beam. The fairing has a slot to accommodate the wing, which is attached to the flexible beam at four locations such that wing flexure and beam flexure are coupled.

The model is instrumented with accelerometers, strain gauges, and pressure ports (Fig. 2). There are 26 vertical accelerometers on the wing and 4 on the flexible fuselage beam. Each engine nacelle has 2 vertical and 2 lateral accelerometers, bringing the total to 38. Strain gauges were located on both the upper and lower surfaces of the wing at 3 locations, oriented to measure both bending and torsion. The control laws that were tested used only accelerometers for feedback, but the effectiveness of the GLA control laws was evaluated using the strain gauges. The model was fabricated with 53 upper surface 40 lower surface unsteady pressure transducers, but they were not used in control-law design or evaluation, and were not included in the simulation. A Videogrammetric Model Deformation (VMD) system ${ }^{14}$ for tracking the motion of targets mounted on the model was employed during testing, but was not used for control-law development or evaluation and was not included in the simulation.

\section{Tunnel Conditions and Vehicle Configurations}

\section{A. Three Wind-tunnel Tests}

The S4T was tested three times in the TDT ${ }^{\pi}$. The tests were conducted in heavy gas, at sub-atmospheric pressures, in order to more closely represent at tunnel scale the dynamic response of a vehicle at full scale. The first two tests were primarily to characterize the model response at various Mach numbers $(M)$ and dynamic pressures $(\bar{q})$, and the third test included testing of RQE, GLA, and FSS control laws. Test conditions for the control laws were focused on three Mach numbers to represent subsonic, transonic and supersonic conditions ( $M=[0.80,0.95,1.1]$ ). For each case, tests were conducted at constant $M$ starting at low $\bar{q}$, and increasing $\bar{q}$ by bleeding in additional heavy gas and adjusting fan speed accordingly. The more recent linear models used in the simulation were generated for the three Mach numbers and a range of dynamic pressures.

\section{B. RCV Resized}

The original RCV for the $1^{\text {st }}$ wind-tunnel entry was found to have very limited control effectiveness. A new RCV was fabricated with surface area three times larger, leading to the designation of $1 \mathrm{xRCV}$ and $3 \mathrm{xRCV}$ to distinguish between the two. The $2^{\text {nd }}$ and $3^{\text {rd }}$ wind-tunnel entries used the 3xRCV exclusively, and only the $3 x R C V$ is represented in simulation.

\section{Ballasted Configuration}

The model was fabricated with axial sleeves in the circumference of the engine nacelles to accommodate addition of metal rods for inertial ballast. The ballast was designed to lower the open-loop flutter speed relative to the nominal, non-ballasted case, in order to bring it more within the operating envelope of the TDT. The intent was also to slow the onset of flutter when the flutter boundary was reached. Engine pylons of different stiffness were fabricated, with similar purpose. Several options were evaluated for ballasting and pylon stiffness, but only one was selected for testing. The two configurations that were tested were therefore designated as Nominal and Ballasted. Each Ballasted nacelle weighed 2.5 times the Nominal value, and the pylons for the Ballasted configuration were only $75 \%$ as stiff. State space models were generated for both the Nominal and Ballasted configurations and were incorporated into the simulation. Only the Ballasted configuration was used during the $3^{\text {rd }}$ tunnel entry for testing feedback control laws.

\footnotetext{
" Specifically tests T597, T600, and T608.
} 


\section{Sample Rate}

The S4T flutter frequency is around $7 \mathrm{~Hz}$, with some potential for adverse interaction with feedback control laws up to about $20 \mathrm{~Hz}$, so a data acquisition system sample rate of 500 samples per second (Hz), and therefore a Nyquist frequency of $250 \mathrm{~Hz}$, was deemed to be adequate to characterize the dynamic response of the model for the $1^{\text {st }}$ windtunnel test. (Signal strength must typically be rolled off at or below the Nyquist frequency in order to have an accurate representation of the system dynamics.) During the $2^{\text {nd }}$ test, it was noticed that frequency responses for wind-off ground vibration tests (GVTs), in still air, tended to exhibit harmonics of the frequencies used to drive the shaker. Between the $7^{\text {th }}$ and $8^{\text {th }}$ run of the $2^{\text {nd }}$ test, the sample rate was increased to $1000 \mathrm{~Hz}$ in order to determine whether the linearity of the responses would improve. The increase in sample rate had no appreciable effect on estimates of model dynamic characteristics or on the GVT harmonics. However, the $1000 \mathrm{~Hz}$ sample rate was retained for the duration of the $2^{\text {nd }}$ test and for the entire $3^{\text {rd }}$ test. After the 3rd test it was determined that the shaker used for the GVTs was the source of the spurious harmonics.

The simulation uses a fixed-step $4^{\text {th }}$ order Runge-Kutta integration algorithm representing a (non-realtime) $1000 \mathrm{~Hz}$ sample rate, in order to match the test conditions. The simulation was run using continuous state-space representations for the plant models, and discrete state-space representations for the control laws. The specification for the format for control laws received from developers required that they be delivered as continuous state-space representations. The control laws were subsequently discretized using a Tustin transformation, for a $1000 \mathrm{~Hz}$ sample rate, for use in both the wind tunnel and the simulation. Because the control laws were delivered in continuous format, the discretization time step could be determined after delivery.

\section{E. Control Surface Excitation}

Data were collected for both response to ambient turbulence conditions and response to control surface excitation. Control surface excitations were used for system identification during each of the tests, and for open-loop CPE during the $3^{\text {rd }}$ test. The $1^{\text {st }}$ tunnel test used both white noise and linear sine sweep excitation for system identification. Post-test analysis indicted that, for the 30- and 60-second sample sets using white noise excitation, the frequency content was non-uniform for a given run and results were not repeatable from run to run. Simulation confirmed that 2- and 3-minute sample sets with clock generated random number seeds showed more variability than was expected. It was found that 6 runs of 30 seconds each, with clock generated random number seeds, established a more repeatable mean than a single run of 3 minutes. Multiple short data sets also provided statistics on variability that were not available with a single, longer run. The linear sweeps produced results with more uniform frequency content and more repeatability. For the $2^{\text {nd }}$ and $3^{\text {rd }}$ tests, logarithmic sine sweeps were used as the primary excitation type, since they dwell longer at the lower frequencies compared to the linear sweeps and therefore provide more low frequency cycles for the limited signal duration. The higher frequencies tend to have many cycles for a given excitation duration for both linear and logarithmic sweeps. The $1^{\text {st }}$ test included data sets with white noise applied simultaneously to 2 or 3 control surfaces. Although the white noise should provide orthogonal (non-interacting) excitation for the multiple channels, the non-uniform frequency content and nonrepeatability masked any orthogonality properties in post-test analysis.

\section{F. Actuators}

The HT and RCV both used linear displacement hydraulic actuators mounted to the flexible fuselage beam. The actuators rotate the control surfaces by means of a lever arm on each respective control surface shaft. The space available for the FLAP actuator was more constrained, and a pivoting vane hydraulic actuator was used. Limits of travel were enforced for the control surfaces, as \pm 12 deg. for the HT and RCV, and \pm 2 deg. for the FLAP. Parameters for $3^{\text {rd }}$ order linear models were estimated for each control surface in order to match frequency responses based on measured data. Parameter values for rate limits were not determined experimentally. Time histories of control surface deflections indicated a time delay non-linearity of about $6 \mathrm{msec}$, or 6 time steps at the faster of two sample rates used for testing. It was not determined what portion of the delay was computational and what portion was related to freeplay or hysteresis. The bandwidth of the actuator for the HT for the $2^{\text {nd }}$ test was found to be very limited. Since the HT was the primary surface used for feedback control laws, a decision was made to exchange the actuators for the HT and RCV prior to the $3^{\text {rd }}$ test. That was a wise choice as it was determined during the $3^{\text {rd }}$ test that not only was the faulty actuator unable to respond well to commands below a threshold amplitude (e.g. 0.5 deg.), the response was also highly load dependent and the faulty actuator was unable to generate sufficient bandwidth with the RCV under aerodynamic load to be useful even for system identification excitation for the $3^{\text {rd }}$ test. The HT actuator (after the exchange) performed adequately for feedback control, although the installed performance when driving the larger HT control surface was not determined prior to closed-loop testing. In the simulation, parameters for the original 'good' actuator were applied to the HT actuator with no adjustment for the 
larger load. Parameters for the RCV actuator were not modified to represent the faulty actuator because amplitude and load dependencies were not modeled. So the simulation models a useable actuator for the RCV even though the one currently installed is only suitable for static trim deflections.

\section{Simulation Functionality}

The simulation was used to support several aspects of the control-law development and wind-tunnel testing. It served to reduce the risks of wind-tunnel testing, and to improve the chances for successful control-law demonstration. It also provided a means to verify the effectiveness of time history analysis tools.

\section{A. Verify Channels, Units, and Signs}

A key benefit of the simulation was to provide an interface for the delivered control laws that was standardized to match the wind-tunnel conventions in terms of channel assignments, units, and sign conventions. The two controllaw design teams had their own linear simulations for control-law development, which initially had non-standard interfaces between plant models and control laws. The first step when receiving linear plant models from the two developer teams or from NASA was to enforce sensor and control surface channel assignments, convert accelerometer units to g's and control surface units to degrees, and ensure that wing accelerometers were positive down, fuselage accelerometers were positive up, and nacelle accelerometers were positive up or starboard depending on whether they had vertical or lateral orientation. With those conventions enforced on the plant models, the control laws also had to be consistent in order to work properly. Of the 38 accelerometers on the S4T, a subset of 18 was agreed upon to be available for feedback control. Thirteen control laws were actually tested, which used a total of 4 of the accelerometers and the HT and FLAP control surfaces. An additional RQE control law was designed for using the RCV control surface but was not considered for testing due to poor performance of the RCV actuator. The RCV control law was designed using the NASA-provided plant models, and used two fuselage vertical accelerometers that were different from the four sensors used by the control laws that were actually tested.

\section{B. Closed-Loop Control RMS}

Another use for the simulation was to give an indication whether control laws would have a tendency to exceed the linear range for control actuators during closed-loop operation in the presence of simulated turbulence. The presence of the turbulence as a continual disturbance is important for a closed-loop stable system because otherwise the control effort will tend toward zero. Control-law gain at the frequency of interest is generally designed as a careful tradeoff between gain and phase margins, or their equivalent, but large control surface activity may be an indication of insufficient control-law roll-off at high frequency or insufficient control-law wash-out at low frequency. Some control laws were observed in simulation to have high gain at low frequency, which was verified in the wind tunnel with CPE analysis that led to modification prior to closed-loop testing.

There were three types of turbulence models available for the simulation, with comparable but not identical spectral and Root Mean Square (RMS) intensity characteristics. Two turbulence models were implemented as $2^{\text {nd }}$ and $3^{\text {rd }}$ order discrete state space models and the third model was implemented as a discrete convolution ${ }^{7}$, with a (half second) impulse function time history to characterize the frequency content.

\section{System Identification for Known Plant}

One advantage of having a simulation along with wind-tunnel testing is having the ability to turn on and off certain aspects of the simulation in order to determine their impact on results, and having a known plant for comparison with system identification estimates based on analysis of input and output time histories. The linear state space models on which the simulation is based can be used directly to generate analytically based frequency responses for reference. Alternatively, time histories can be generated for sensor responses due to commanded control surface excitation, and frequency responses then estimated from those time histories. The frequency response estimates are influenced by choices concerning things such as amplitude and frequency content of the excitation signal, duration of the time record, windowing type, block size, and overlap for the signal processing. Also, assumptions behind the linear analysis techniques are violated to some extent when non-linearities such as time delays, and rate and position limiting of the actuators are present.

Results can be compared to the analytical reference results in order to develop confidence levels for how well the actual plant characteristics are estimated from time history data. The simulation allows runs to be repeated with and without the presence of the non-linearities in order to observe the degradation of the estimated results. Likewise the non-measured turbulence input to the plant model can be turned on and off to determine its impact. Artifacts of the 
estimation process identified through the use of simulation can then be recognized if present when processing time histories from wind-tunnel tests.

With turbulence active, multiple runs can be made with clock-generated random number seeds for driving the turbulence in order to characterize the amount of variability from one run to the next. Such confidence levels and variability characterizations can be useful for control design techniques that explicitly take uncertainties into account. It is of interest to note that what is observed in the wind tunnel and described as "vehicle response to bursts of turbulence" are observed in simulation for cases where the turbulence itself is uniform. Apparently the bursts of turbulence are really "bursts of vehicle response in the presence of uniform amplitude turbulence" that are more closely related to sympathetic phasing of the turbulence relative to the vehicle vibrations. That observation would be difficult to make in the wind tunnel without some means of measuring the actual turbulence.

\section{Conditions Not Tested In Wind tunnel}

The three wind-tunnel tests so far have only been conducted at conditions below the open-loop flutter boundary. However, one case of interest is to identify crossing the open-loop flutter boundary when operating closed-loop. The simulation provides a means for obtaining time histories representative of a case that is artificially stabilized through feedback in order to determine qualitatively the reliability of estimates of the open-loop boundary in the presence of turbulence and actuator non-linearities.

Another case for which there is no S4T wind-tunnel data is for closed-loop testing of a control law that uses more sensors than controls for feedback. Analysis is typically done by exciting individually each control surface used for feedback and recording responses on all feedback sensor channels. For the case where there are more feedback sensors than controls, not enough information is generated by control excitation to be able to distinguish the contribution of each individual sensor. Either the excitation needs to be injected into each sensor channel, or the control law itself must be considered to be a known component of the system. ${ }^{6}$ Verification that a control law is implemented as intended is an important part of wind-tunnel testing, but whether the control law is considered to be known or whether excitation is added to the sensor signals, the processing of a feedback control law with more sensors than controls is different from that used for any S4T control laws tested to date, so the simulation us useful for verification of analysis software.

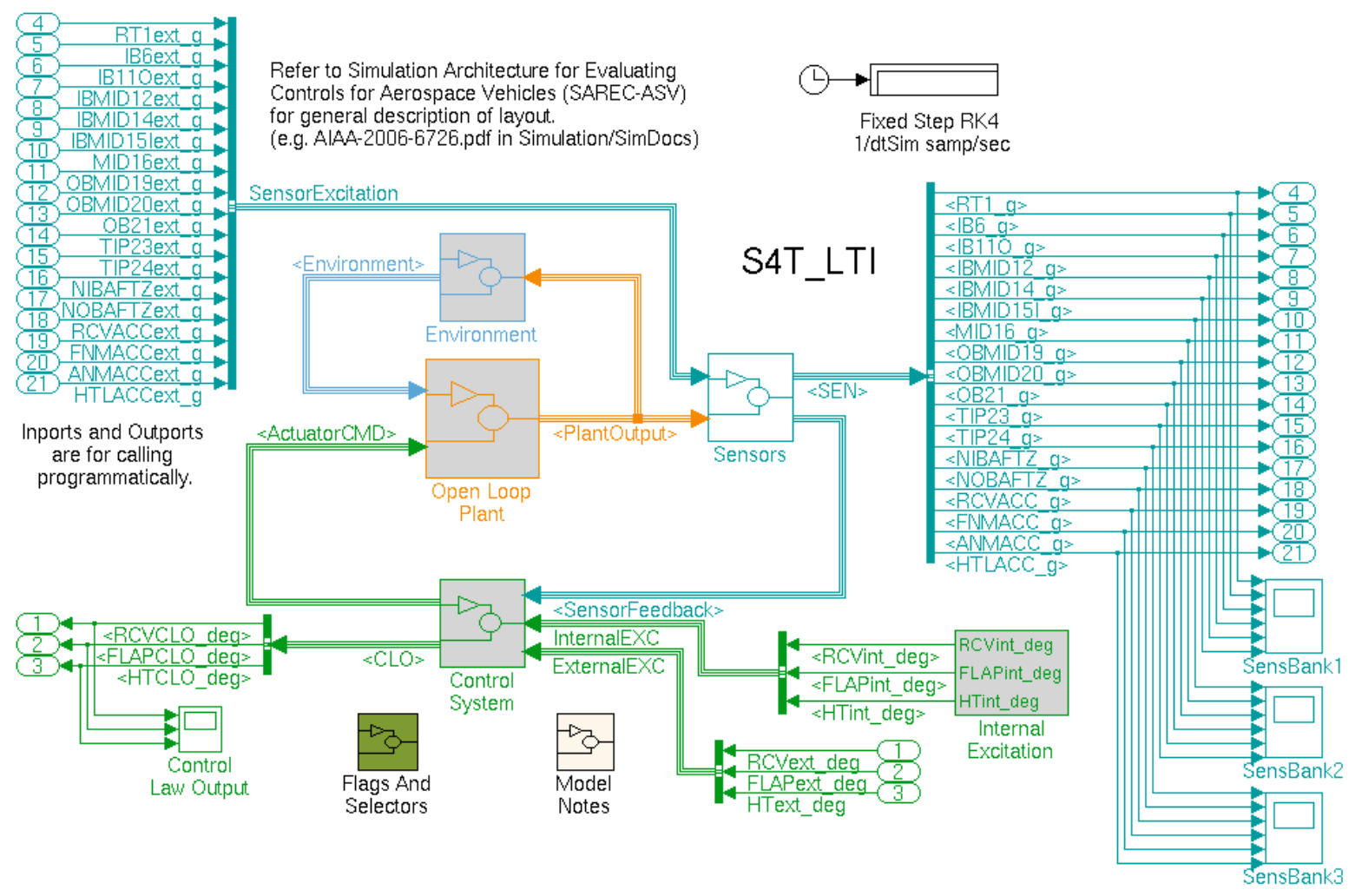

Figure 3. SAREC-ASV Top Level Diagram. The Controls, Sensors, Environment, Open Loop Plant, Inports, and Outports. 


\section{Simulation Implementation}

A "small disturbance" justification for the validity of using linear state space models for representing S4T vehicle dynamics was generally satisfied due to constraints imposed by the mounting system. Several MATLAB and Simulink constructs were used to systematically incorporate state space plant models for multiple test conditions ( $M$ and $\bar{q}$ ), provided by different design teams and representing various vehicle configurations.

\section{A. Linear Time Invariant (LTI) Objects}

Equations of motion for the S4T were generated using NASTRAN for the structural model and using various linear and non-linear analysis codes for unsteady aerodynamics. Some later models were adjusted based on Ground Vibration Test (GVT) data obtained in air at zero dynamic pressure. The result was a series of linear models for various $M$ and $\bar{q}$ combinations for various S4T configurations and methods for unsteady aerodynamics analyses. In order to incorporate the linear models in a systematic way, groups of linear models were modified for conformity with wind-tunnel conventions, and then loaded into MATLAB LTI objects.

LTI objects contain data that is accessible as though contained in a MATLAB hierarchical data structure (Table 1), but they also define certain functionality associated with the transfer function, pole-zero, state space, or frequency response models contained within them. LTI syntax permits access to rows and columns of the state space ABCD type matrices using a 2-dimensional array notation, but also allow the notation to expand to represent multiple models. For the S4T, a $3^{\text {rd }}$ index was assigned to represent $M$ and a $4^{\text {th }}$ index was assigned to represent $\bar{q}$. The largest single LTI object represented 6 Mach numbers and 51 dynamic pressures, for a total of 306 flight conditions. The LTI objects have standardized fields as shown in the upper portion of Table 1. For example, the Notes field contained information about the origination date for the models, a description of techniques used in generating the models, and a point of contact for finding out more information. Although UserData itself is a standard field, users can add subordinate fields to UserData. If an LTI object is duplicated, all the standard fields are replicated except for the UserData field, which must be copied as a separate step. For the S4T, UserData was used to store input and output signal units, signs, and descriptions. Two items in the UserData field that are required for running simulation scripts that initialize the plant model are vectors that define the $M$ and $\bar{q}$ conditions represented by the state space models in the LTI object.

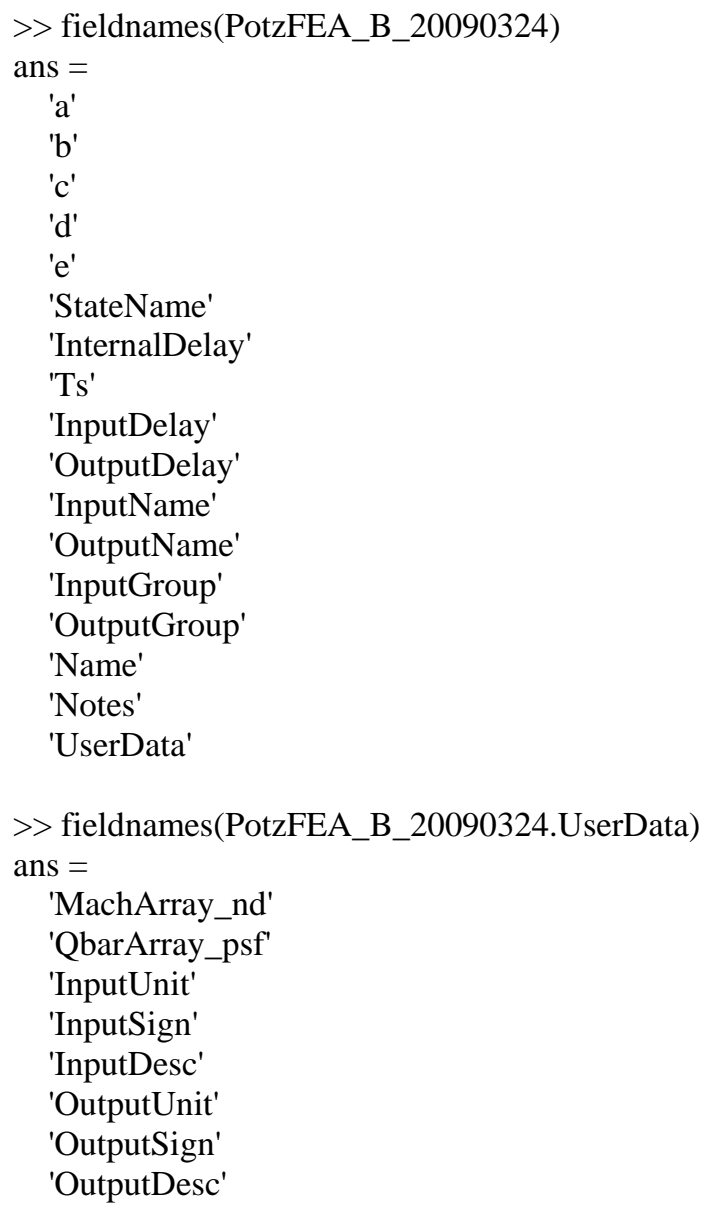

Table 1. LTI Object Fields. Names for standard fields of a MATLAB LTI object, and for user-defined S4T fields in UserData.

\section{B. Configurable Subsystems}

Each LTI object implemented in the simulation contains state space models for various $M$ and $\bar{q}$, but for a single RCV size, ballasting, design team, and modeling technique for the unsteady aerodynamics. In order to host several different LTI objects, a Simulink Configurable Subsystem capability was used to define a template block for the plant model that can represent any of the various LTI objects. Figure 3 shows the top level Simulink diagram for the S4T_LTI simulation. The Controls, Sensors, and Environment blocks are external to the Open Loop Plant. Top level Inports and Outports (Fig. 3) define function arguments when the simulation is run from a script. Inside the Open Loop Plant block is a TEMPLATE block which defines a common set of inputs and outputs for Simulink blocks in a User Defined Library that each host an LTI object (Fig. 4). The Environment input to the TEMPLATE 


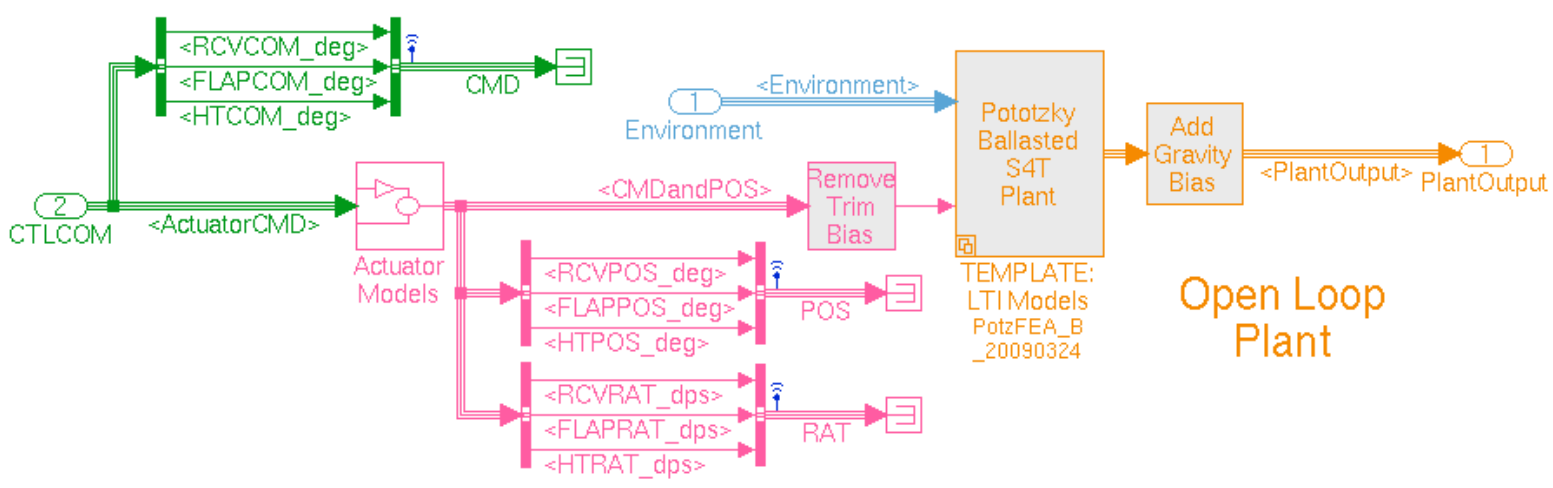

Figure 4. Template Block. TEMPLATE block for various User Library blocks that each host an LTI object, and 'beacons' for the CMD, POS, and RAT busses.

block provides turbulence rate and acceleration for plants that respond to external turbulence, and white noise for plants with an internal turbulence model. The controls input to the TEMPLATE block provides control surface positions (POS), rates (RAT) and accelerations (ACC) for plants that respond to external actuators, and actuator commands (CMD) for plants with internal actuator models.

The 'beacons' on the CMD, POS, and RAT busses log those signals as simulation output that supplements the top level Outport signals. The simulation was implemented with biases on control surfaces and sensors in order to verify that analysis routines for plant estimation and RMS calculations properly account for non-zero mean values. Color is used to distinguish commands as green, control surface positions, rates, (and accelerations, not shown) as magenta, Environment variables as blue, and state variables as burnt orange.

\section{Actuators}

Actuators were implemented in the Actuator Models block as having a third order linear component, plus a time delay and rate and position limits (Fig. 5). A generic actuator was placed in a Simulink library, with a mask used to define parameter values specific to each control surface when the library block was used in the simulation. An effective time delay was observed in the experimental data, and was implemented with a value of 6 msec as the Delay block where the command signal enters the actuator model. Rate and position limits were imposed using Simulink limited integrators with additional logic to ensure that acceleration integrators were reset to zero when rate limits were reached, and both acceleration and rate integrators were reset to zero when position limits were reached. For example, control surface acceleration should not be allowed to drive the inertial "tail wags dog" effect while a control surface is on a position limit. Saturation ports on the position and rate integrators were used to indicate whether an upper or lower limit was reached.

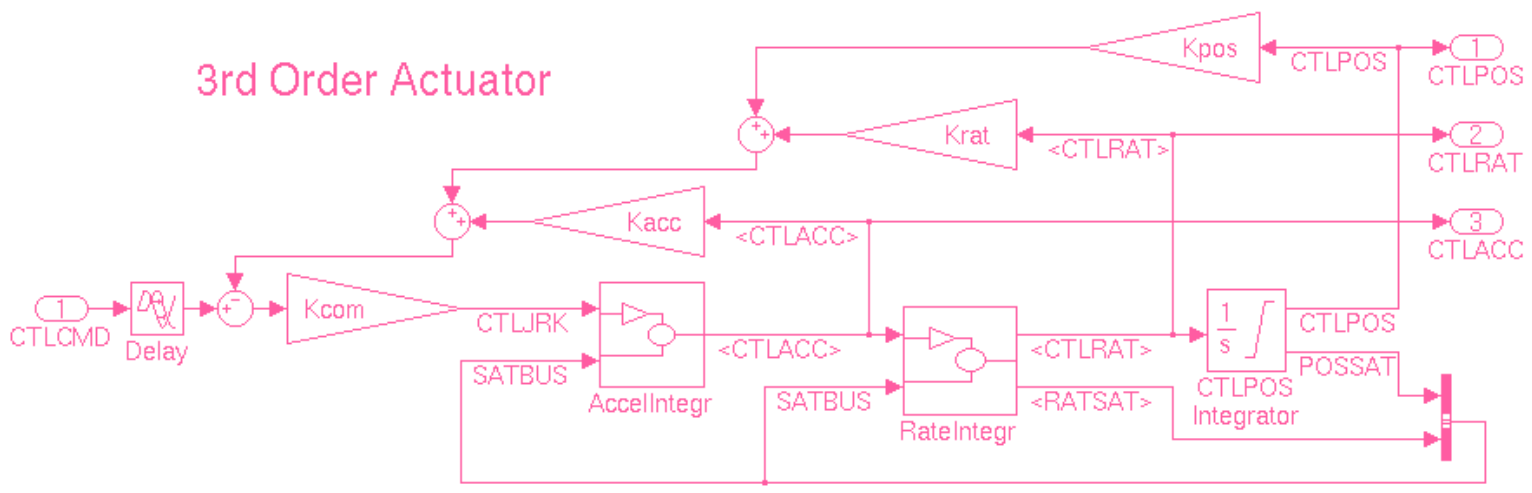

Figure 5. Actuator Library Block. $3^{\text {rd }}$ Order Actuator model located in a Simulink User Library, with Simulink Mask for setting parameter values.

American Institute of Aeronautics and Astronautics 


\section{Control Laws}

Thirteen control laws out of 94 submitted were actually tested in the wind tunnel. The ones tested were each Single-Input/Single-Output (SISO) or two-input/two-output (MIMO, for Multi-Input/Multi-Output). The most prevalent combination for SISO control used an inboard nacelle aft vertical accelerometer to drive the HT control surface. In all, both HT and FLAP were used in combination with a subset of four accelerometers. Control-law complexity varied from $4^{\text {th }}$ order to $21^{\text {st }}$ order.

Ground rules drafted for the format of control laws delivered for testing specified that the control laws were to be provided as continuous state space models. Each control law was then discretized using a Tustin transformation at $1000 \mathrm{~Hz}$, and padded to 18 sensors, 3 controls, and 40 discrete state variables. The padding was used so that each control law could be loaded into the wind-tunnel control computer in specific memory locations after the controller software was compiled with the specified dimensions. The intent was to have the hand-off between control-law designers and control system implementers to be as narrow and explicit as possible, rather than have each controllaw designer responsible for discretization and padding.

Each control law was to be provided in a separate data file, with no packing of multiple control laws into LTI objects. A naming convention was established for control-law files and for the state space control laws in each file. The convention used the designer's identifier, an identifier for the type of control provided (e.g. FSS or GLA), a designation of ' $\mathrm{B}$ ' for use with the ballasted configuration, $M$ and $\bar{q}$ for which the control law was designed, and a version identifier. The state space object User Data field was to contain Point Of Contact (POC) information, a release date, and indication of sensor and control command units and sign convention.

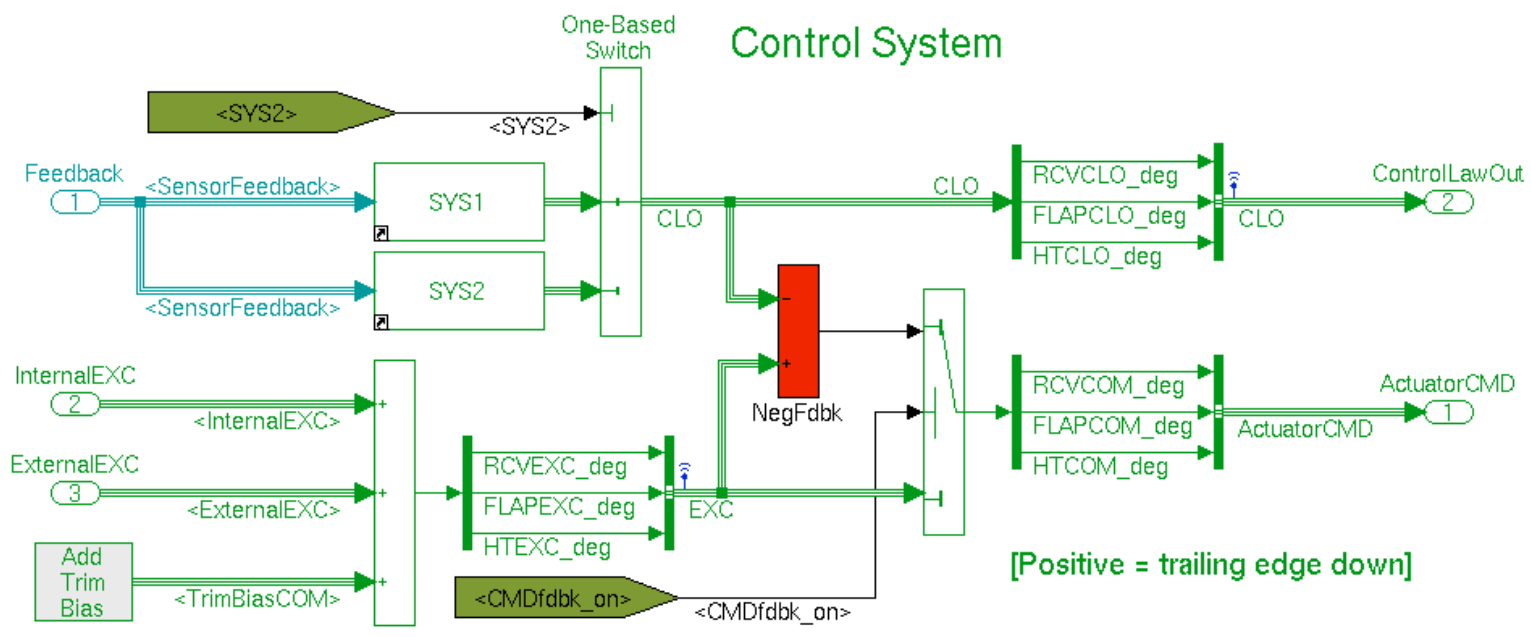

Figure 6. Feedback Control Junction. Internal and external excitation, generic SYS1 and SYS2 controller blocks, feedback loop closure, and beacons for EXC excitation and CLO Control Law Output.

Control-law designers were to provide frequency responses representing the intended control-law dynamics for verification against estimates based on time histories generated after loading a control law into the controller computer. The designs were to accommodate the discrete sample-hold (finite time resolution), quantization (finite numerical resolution), and time delay effects associated with the digital implementation. The highest number of controls and sensors used by candidate control laws was 3 controls and 8 sensors (although that was not tested in the wind tunnel). MIMO control laws were to be evaluated in terms of singular values of a return difference matrix at both the controls (e.g. 3x3) and at the sensors (e.g. 8x8), depending upon how many were used for feedback ${ }^{6}$. The singular values were estimated as a function of frequency, and small values at a particular frequency indicate a potential for instability at that frequency as dynamic pressure is increased. Singular values were estimated both in simulation and experimentally, based on analysis of time histories.

\section{E. Control Surface Excitation}

Simulation control excitation can be generated internally using Simulink signal generating options, or externally by means of a calling script and the top level diagram Inports. Excitation is the sum of those two sources, plus a trim bias (Fig. 6). Excitation plus feedback gives the total command, on the CMD bus. Signals on the bus are labeled 
COM for compatibility with the wind-tunnel signal names, but CMD is used for the bus to distinguish from 'Common' and 'Communications'. The feedback loop is opened or closed using a flag. The feedback summer has a negative sign, by convention. A similar summer for feedback at the sensors has a positive sign, so care must be exercised with regard to sign when breaking the loop at controls or sensors for a MIMO control law. Two control laws are loaded and run simultaneously as SYS1 and SYS2. Selection of control law for feedback is made using an index of 1 or 2. There is a beacon for logging both EXE (Excitation) and CLO (Control-law Output) as simulation output.

Excitation signals are generally chosen based on frequency content and amplitude. Logarithmic sine sweeps allow visual interpretation based on variation in amplitude of response as the excitation sweeps through various frequencies. However, it can be limited in amplitude at all frequencies based on a large vehicle response in a lightly damped but narrow frequency range. Random or pseudorandom excitation avoids having a peak amplitude response as a specific frequency is traversed, but cannot be as easily interpreted by visual inspection, and in the case of white noise driven signals, may lack repeatability and uniformity of frequency content.

\section{F. Turbulence Models}

Three turbulence models run simultaneously in the simulation, with an index used to select which type is sent to the plant model (Fig. 7). Each model generates a vertical turbulence velocity, and a derivative block is used to generate a vertical acceleration. The turbulence models take white noise as input, each from the same noise source so that plots of the turbulence can be compared directly. Two turbulence models are state space models of $2^{\text {nd }}$ or $3^{\text {rd }}$ order. The coefficients for a continuous representation of the state space ABCD matrices are defined in a Simulink mask for each model, with parameter dependencies for $M$ and $\bar{q}$. The Mask also interrogates the simulation to determine the fixed-step sample rate, and discretizes the model using a Tustin bilinear transformation. The third turbulence model uses a discrete convolution representation that is able to match a non-rational slope for the high frequency roll-off of the power spectrum ${ }^{7}$. A flag is used to choose either a clock-generated seed for the random number generator, or zero as the seed in order to have a repeatable check case.

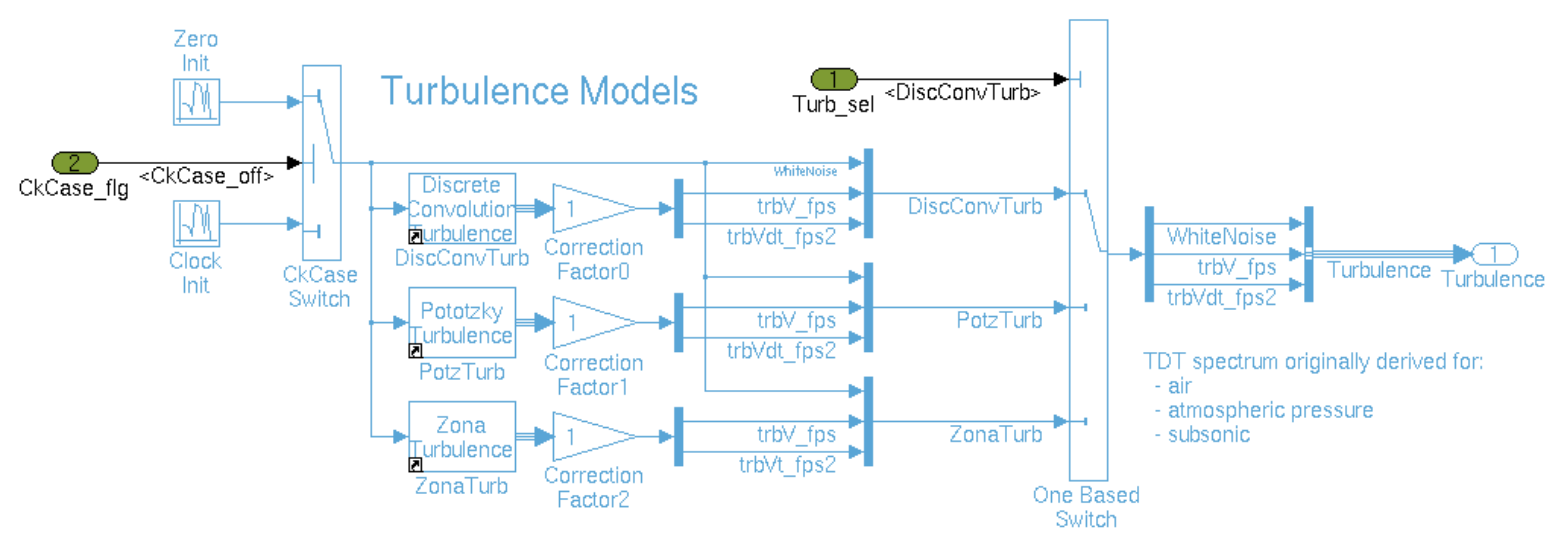

Figure 7. Turbulence Models. Three turbulence models with index to select type, and flag to select clock-based or zero for random number seed.

\section{G. Graphical User Interface}

The S4T GUI is simplified compared to earlier SAREC GUIs in that is it not reconfigurable for finding different types of trim conditions, and it has only one window rather than having a family of interrelated windows. GUI functionality for loading different plant models or control laws is provided by running MATLAB scripts that obtain user information from the MATLAB command line rather than by calling additional GUI windows. Information about the loaded plant, the tunnel conditions ( $M$ and $\bar{q}$ ), and two loaded control laws is displayed on the GUI (Fig. 8). If plant model or control laws are changed by means other than the GUI, clicking on the GUI background will update the display to show the current status. The GUI can be used to Start, Pause, Resume, and Stop a simulation run. Flag settings can be changed during a run for turning feedback on or off, switching between the two loaded control laws, activating control surface excitation, or turning turbulence on or off. Switching during a simulation run allows assessment of transient behavior, but requires saving time-based information about the 
switching in order to fully characterize a simulation run. The GUI also permits selection of the type of surface excitation, and type of turbulence, although selecting an item from a menu requires reinitialization of the simulation to start a new run. The simulation duration can be set from the GUI, as well as selecting a standard random number seed for a repeatable random number sequence for turbulence. The GUI can be used to open or close a set of Simulink scopes for display of accelerometer and control surface time histories, and can open the Simulink diagram itself. Finally, the GUI can initiate saving the time history data in a wind-tunnel format that is compatible with windtunnel data analysis tools.

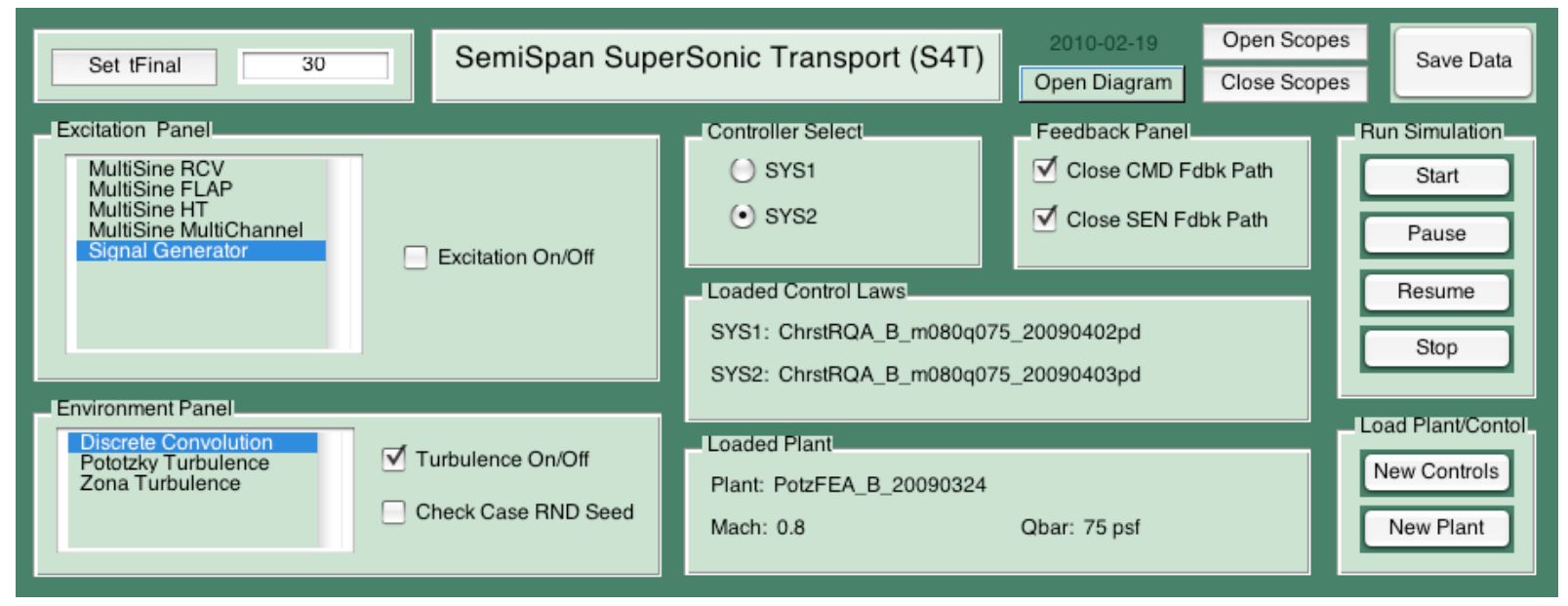

Figure 8. Graphical User Interface. S4T GUI used to load new plant models and control laws, choose type of control surface excitation and turbulence, set run duration, control execution, and change settings during a run.

The GUI interacts with settings for the Simulink diagram through a narrow interface. The simulation uses flags (binary) and selectors (integers) that are defined in the "Flags And Selectors" subsystem block on the top-level diagram (Fig. 3). The values of the flags and selectors are applied at the various locations where they are used in the simulation by means of Simulink To/From Tag blocks. (The tags act somewhat like 'GOTO' statements, so they should be used judiciously to avoid developing a Simulink analog to FORTRAN spaghetti code.) The signal wires carry the values of the flag settings, but SAREC assigns a name to each flag and selector signal that indicates the value of the signal. The tag blocks propagate the name of the signal that is passed through, so the status of a switch can be determined by looking at the name of the signal that drives the switch.

\section{Characteristics of Linear Models}

Linear state space models are generally used for modeling small amplitude aeroelastic motion. The small amplitude motion can be superimposed on large amplitude six degree-of-freedom rigid body motion to give a free flight aeroservoelastic model. ${ }^{2}$ In the case of the wall mounted S4T, the rigid body degrees-of-freedom are restricted such that the state space models represent nearly the entire mathematical model of the vehicle. State space models can be used directly to generate open and closed-loop linear time histories without a Simulink host. SAREC provides a way to realize the simulation in a form that replicates some nonlinearities present in the physical model, but the state space models are of central importance. Some aspects of the NASA-provided state space models that have an impact on implementation in simulation are discussed below.

\section{A. Actuators and Turbulence External to Plant}

Linear models for actuators can be incorporated with linear models of the vehicle dynamics. Likewise, if turbulence is modeled as a state space filter for white noise, the turbulence model can also be incorporated into the plant model. Using that approach, inputs to the plant model would be commanded control deflections for control excitation, and white noise for turbulence. If the plant model is then subjected to order reduction through residualization, then the state variables for the actuator models and for turbulence get combined with state variables for the vehicle aeroelastic (and generally rigid body) degrees-of-freedom such that it becomes difficult to extract the actuator and turbulence models. A disadvantage to that approach is that if the characteristics of the actuators change, the plant model cannot readily be updated to incorporate the change without going back to an earlier version of the 
plant model. Also, actuator rate and position limits can't be enforced directly. Limits can be imposed on the commanded deflections, but the internalized actuator models will not be constrained against overshoot. Likewise, an internalized turbulence model is difficult to compare against spectral characteristics estimated from physical measurements. The NASA-provided plant models had inputs for control surface positions, rates, and accelerations that permitted updates to actuator models and imposition of limits by having the actuator models external to the plant. The plant models also had inputs for vertical turbulence rate and acceleration such that the characteristics of the turbulence models could be evaluated separately from the plant models, and the discrete convolution turbulence model could be used as well as the state space turbulence filters for white noise.

\section{B. Uniform Test Grid and Number of State Variables}

Incorporating groups of state space models into LTI objects was facilitated by uniformity in $M$ and $\bar{q}$ gridding and in the number of state variables used for each model. Wind-tunnel tests were generally conducted at constant Mach number and increasing values for dynamic pressure, in order to not have Mach number related changes in flow characteristics for a given set of runs. Having state space models available for constant $M$ and increasing $\bar{q}$ was therefore consistent with operational procedures. Having consistent values for $\bar{q}$ for each of the Mach numbers for which plant models were provided allowed incorporating all models into a single LTI object that was parameterized by an index for $M$ and an index for $\bar{q}$. Otherwise a separate LTI object would have been needed for each Mach number in order to provide the required coverage.

Having the same number of state variables for each plant model made it easier to "connect the dots" when plotting a root locus based on increments in $\bar{q}$. Otherwise some dynamic pressures would have more roots to plot and some would have fewer making it difficult to track the migration of roots. Having a fixed number of state variables, with no residualization to distort the underlying structure of the stability matrix, is also essential if interpolation of the ABCD matrices is to be used to generate models for intermediate values for $M$ and $\bar{q}$ during a simulation run, as was done for piloted simulated landings for the HSCT simulation. ${ }^{2}$

\section{Standardized Channels, Units, Sign Convention}

For candidate control laws there was a specified format that included channel assignments, units, and sign conventions. The standardization allowed new control laws to be loaded directly without customized implementation. The plant models did not have a specified standard, but it was important that they were made to conform to a standard that was consistent with the wind tunnel and control-law conventions. An interesting case arose when an internalized actuator model was extracted from a contractor-supplied plant model. The commands to the actuator were in degrees, but the units for actuator output and for plant input were radians. Prior to actuator extraction the units were internal and consistent, so that was not an issue. After actuator extraction, the interface between actuator and plant needed to be converted to degrees in order to establish the required consistency with other models.

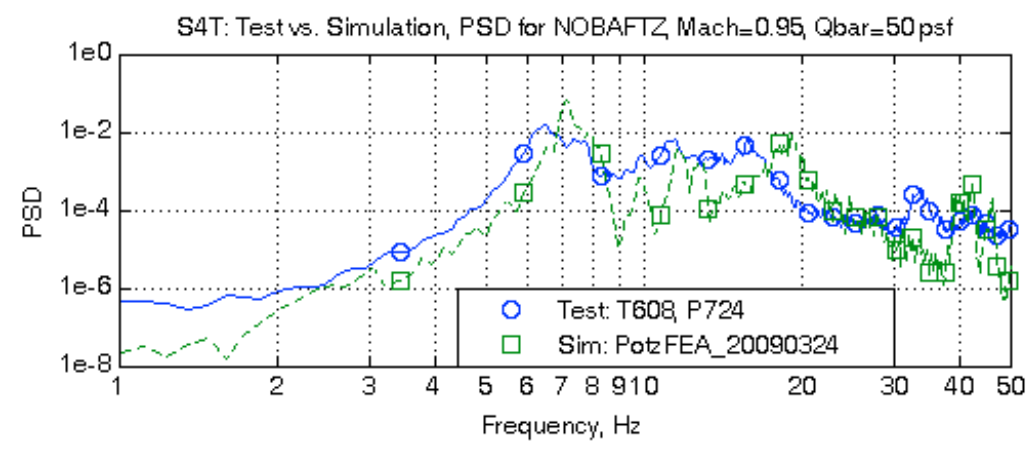

Figure 9. Power Spectral Density. Overlay of Power Spectral Densities estimated from time histories of vertical acceleration at the aft end of the outboard nacelle (NOBAFTZ accelerometer) due to turbulence for the wind-tunnel model and for the simulation. 


\section{Strain Gauges vs. NASTRAN Cutting Planes}

Another consideration arose when comparing loads outputs from the NASAprovided plant models versus the strain gauge measurements available from wind-tunnel testing. The strain gauges were not used by any of the control laws for feedback, but were used for assessing the effectiveness of gust load alleviation. The strain gauges measured strains at specific isolated locations on the airframe, which were a function of load paths in the structure and the dimensions and material properties of the airframe component to which the strain gauges were attached. The NASTRAN finite element models used for the S4T were never intended to accurately represent strain at that level of detail. Experimentally, a bench test calibration was used to relate the strain gauge outputs to specific loading of the S4T wing in bending and torsion. A cutting plane method was applied to the NASTRAN models when generating the state space models in order to calculate the total forces and moments transmitted across the cutting plane. ${ }^{13}$ Otherwise it would not have been possible to provide a GLA load assessment using the simulation. The cutting plane method gives analytical forces and moments at the location of the cutting plane. A distinction to realize is that strain gauges are calibrated for response to external loads such as bending and torsion, but that any single gauge will respond to both bending and torsion, so distinguishing between even those two loading conditions would really require use of multiple gauges.

\section{Simulation Results}

Applying wind-tunnel data analysis techniques to time histories generated by the simulation provides a means to assess the fidelity of the plant estimation process.

\section{A. PSDs for Ambient Conditions}

Power Spectral Density (PSD) analysis is useful for showing overall strength as a function of frequency for a single channel of data. Figure 9 shows a comparison of PSDs derived from wind tunnel and from simulation time histories. In each case the response is due to turbulence, or a model of turbulence, and measurements of the turbulence are considered to be unavailable. The plots are for 0.95 Mach number and 50 psf dynamic pressure.

For the wind-tunnel data, two peaks associated with flutter are located at about $6.5 \mathrm{~Hz}$ and $7.5 \mathrm{~Hz}$. At $50 \mathrm{psf}$ dynamic pressure the two peaks have somewhat coalesced, with the $6.5 \mathrm{~Hz}$ mode becoming the more dominant of the two. As the flutter dynamic pressure is approached the $6.5 \mathrm{~Hz}$ mode moves up in frequency, and instability occurs near $7 \mathrm{~Hz}$. Below the critical dynamic pressure there is also significant response amplitude at $11 \mathrm{~Hz}$ and $16 \mathrm{~Hz}$ with less amplitude at other frequencies.

For a simulation-based estimate of the PSD for the NASA-provided linear model corresponding to the same $M$ and $\bar{q}$, two peaks are evident at about $7 \mathrm{~Hz}$ and $8 \mathrm{~Hz}$, with the coalescence of peaks somewhat more pronounced 

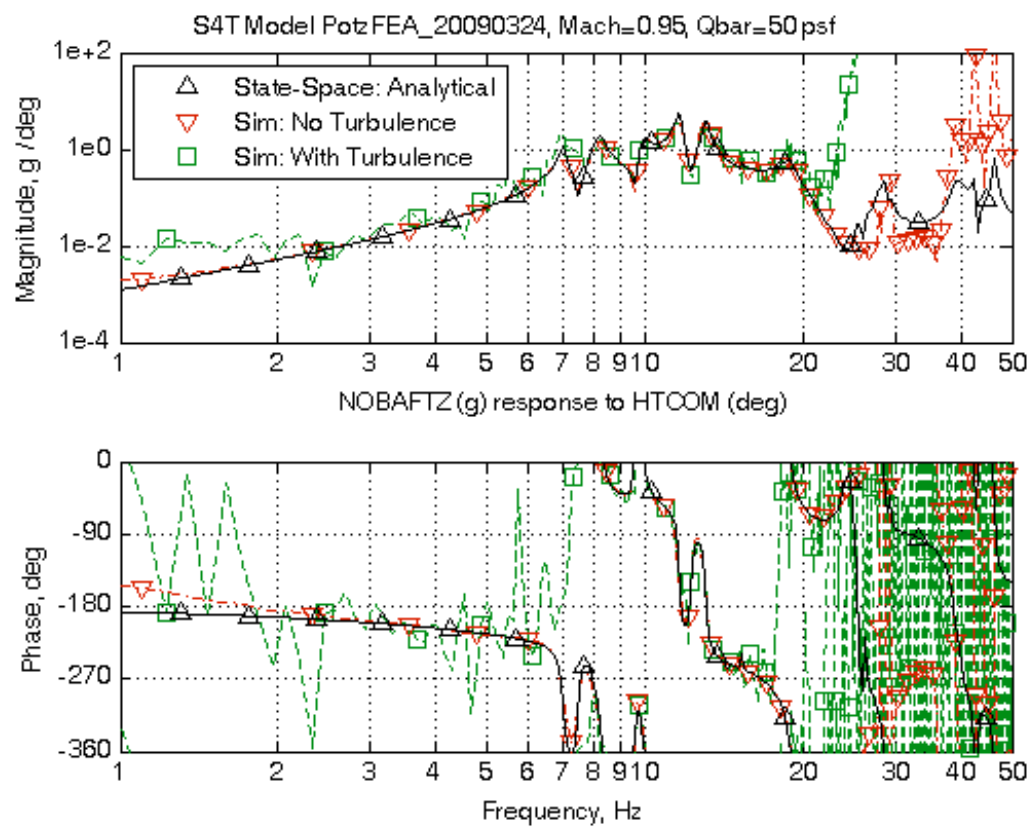

Figure 11. Degraded Frequency Response Estimates. Simulationbased frequency response estimates for NOBAFTZ accelerometer due to HT excitation. than for the wind-tunnel-based result. The experimental $16 \mathrm{~Hz}$ mode appears to be represented in the analytical model at about $18 \mathrm{~Hz}$, with a closer frequency match for the $11 \mathrm{~Hz}$ mode.

The lesser degree of coalescence for the flutter related modes in the wind-tunnel model would suggest that it is somewhat farther from the unknown experimental open-loop flutter dynamic pressure than the analytical model is from the analytical flutter dynamic pressure. Predictions of the critical flutter dynamic pressure for the experimental model, based on analytical models, can be improved by adjusting parameters in the analytical (state-space) models to match results for subcritical tunnel test data.

A flutter suppression control law designed and evaluated based on analytical models may not work well during wind-tunnel testing, depending upon sensitivity to differences in things such as frequencies for peak response. For full scale application, a practical flutter suppression control law may need to be scheduled based on vehicle weight as fuel is burned in order to track changes in modal characteristics and to provide flutter margin on demand whenever transitioning through the transonic regime. Control-law robustness to changes in plant characteristics would reduce the need for scheduling.

\section{B. Frequency Responses for Forced Excitation}

Figure 10 shows time histories for data collected in the wind tunnel for forced response at 0.95 Mach number and $50 \mathrm{psf}$ dynamic pressure. A \pm 0.3 degrees amplitude logarithmic sine sweep excitation from $0.5 \mathrm{~Hz}$ to $25 \mathrm{~Hz}$, as shown in the first time history, was applied to the HT actuator. The measured response for the HT control surface is shown in the second time history. The third time history shows the response of the vertical accelerometer located at the aft end of the outboard nacelle (labeled "NOBAFTZ"). The accelerometer response is dominated by tunnel turbulence for this test condition, such that there is no obvious increase in activity for the accelerometer during the sweep except for the time from 85 to 90 seconds. The frequency of the
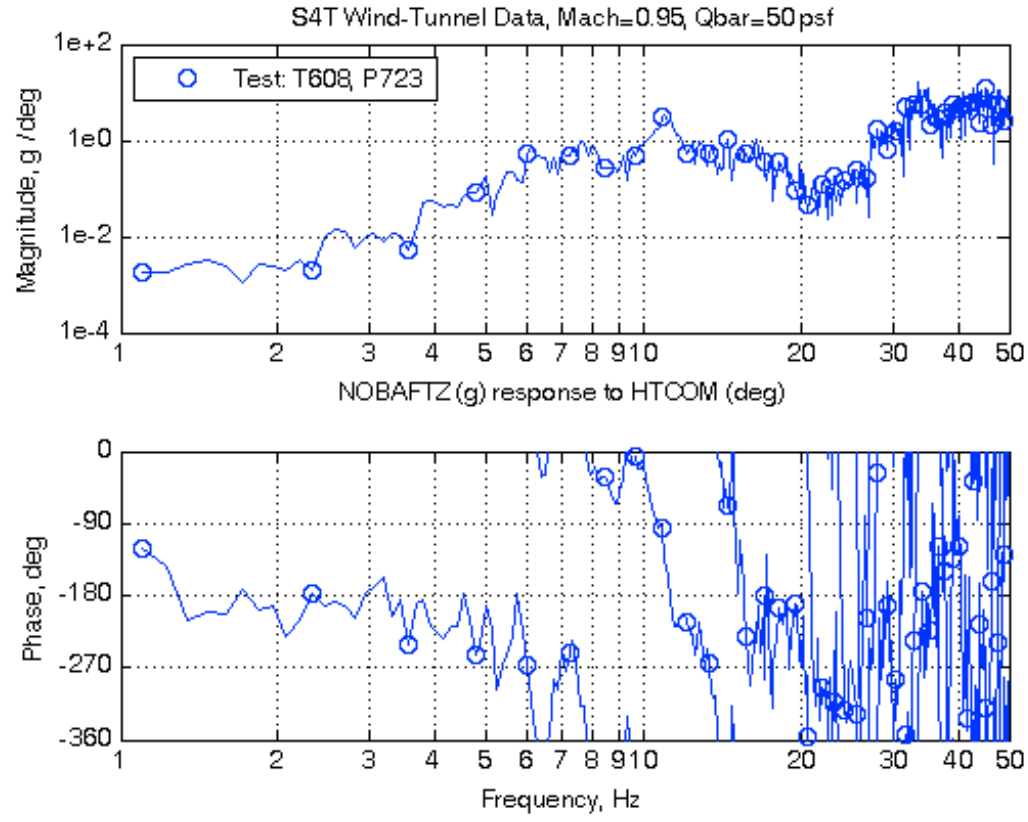

Figure 12. Experimental Frequency Response Estimate. Wind-tunnelbased frequency response estimate for NOBAFTZ accelerometer due to HT excitation, calculated from Figure 10 time histories. 
sine sweep during that time period corresponds to about $11 \mathrm{~Hz}$. The challenge is to estimate the response to the control surface excitation despite the presence of turbulence, without over stressing the model by using excessively large amplitude excitation.

The simulation can be used to assess the impact of poor signal to noise ratio on estimating the plant response. Figure 11 shows an analytically-based frequency response derived directly from the NASA-provided state-space model for the plant at 0.95 Mach number and 50 psf dynamic pressure for the NOBAFTZ accelerometer due to the HT actuator command. That representation is not subject to corruption due to a Nyquist frequency sample rate limitation, excitation signal deficiency, or noise. Also shown are estimates based on simulation time histories run with and without turbulence, again for a 0.3 degree logarithmic sine sweep for the HT. Differences between the estimated and the analytically based curves are artifacts of the estimation process. Qualitatively the estimate generated without turbulence gives a very good match up to $25 \mathrm{~Hz}$, which is the upper limit of the frequency content of the excitation. Above that frequency the estimate degrades, with the amplitude noticeably large above $35 \mathrm{~Hz}$. The amplitude estimate generated in the presence of turbulence is very good from about $2.5 \mathrm{~Hz}$ up to $21 \mathrm{~Hz}$, with degraded phase estimation even in the critical flutter frequency range around $7.5 \mathrm{~Hz}$. Above $21 \mathrm{~Hz}$ the amplitude estimate is much too large and the phase estimate cycles repeatedly through 360 degrees.

Note that the two modes at about $7 \mathrm{~Hz}$ and $8 \mathrm{~Hz}$ are more distinct than they were for the PSD for the same condition, and the amplitude is greater from $10 \mathrm{~Hz}$ to $15 \mathrm{~Hz}$ than from $6 \mathrm{~Hz}$ to $8 \mathrm{~Hz}$, which is also different. The differences are due to the nature of the HT excitation at one fuselage location compared to turbulence applied directly on the aerodynamic surfaces.

Figure 12 shows a frequency response estimate based on the wind-tunnel time histories represented in Figure 10. The large amplitude time history response noted previously in the vicinity of $11 \mathrm{~Hz}$ is evident as a peak at the same frequency in the frequency response plot. The estimated amplitude increases and the estimated phase wraps repeatedly above the $25 \mathrm{~Hz}$ upper limit of the sweep excitation, similar to the simulation result generated with turbulence active. Those magnitude and phase characteristics are likely artifacts of the estimation process, as they are with the simulation.

\section{Concluding Remarks}

SAREC provides a simulation framework that is motivated by reusability. By emphasizing a component-based architecture, much of the simulation can be retained intact when applying SAREC to a new vehicle model. SAREC does not represent an entirely new way of doing things, but offers advantages as a starting point for developing simulations for new vehicle models as compared to the earlier simulations on which SAREC was based.

The SAREC-based simulation of the S4T provides a useful tool for use in conjunction with wind-tunnel testing. Various control laws can be simulated with various plant models without using tunnel time. Analysis tools for estimating plant characteristics and stability boundaries based on time histories can be evaluated against known plant models, with and without turbulence active for corrupting the time history signals. The simulation architecture used supports incorporation of multiple plant models. The GUI provides for opening or closing the feedback loop during a simulation run, or switching to a reversionary control law, in order to assess transients.

\section{References}

${ }^{1}$ Christhilf, D. M., and Bacon, B. J., "Simulink-Based Simulation Architecture for Evaluating Controls for Aerospace Vehicles (SAREC-ASV),” AIAA Modeling and Simulation Technologies Conference, AIAA-2006-6726, Keystone, CO, 2006.

${ }^{2}$ Sotack, R. A., Chowdhry, R. S., and Buttrill, C. S., "High Speed Civil Transport Aircraft Simulation: Reference-H Cycle 1," NASA/TM-1999-209530, 1999.

${ }^{3}$ Stevens, B. L., and Lewis, F. L., Aircraft Control and Simulation, $2^{\text {nd }}$ ed., John Wiley \& Sons, Hoboken, NJ, 2003, Chap. 3, and Appendix A.

${ }^{4}$ Nguyen, L. T., Ogburn, M. E., Gilbert, W. P., Kibler, K. S., Brown, P. W., and Deal, P. L., “Simulator Study of Stall/PostStall Characteristics of a Fighter Airplane With Relaxed Longitudinal Static Stability,” NASA TP-1538, 1979.

${ }^{5}$ Perry, B., III, Silva, W. A., Florance, J. R., Wieseman, C. D., Pototzky, A. S., Sanetrik, M. D., Scott, R. C., Keller, D. F., Cole, S. R., and Coulson, D. A., "Plans and Status of Wind-Tunnel Testing Employing an Aeroservoelastic Semispan Model," 48th AIAA/ASME/ASCE/AHS/ASC Structures, Structural Dynamics, and Materials Conference, AIAA-2007-1770, Honolulu, HI, 2007.

${ }^{6}$ Wieseman, C. D., Hoadley, S. T., and McGraw, S. M., “On-Line Analysis Capabilities Developed to Support the Active Flexible Wing Wind-Tunnel Tests,” Journal of Aircraft, Vol. 32, No. 1, 1995, pp. 39-44.

${ }^{7}$ Pototzky, A. S., and Christhilf, D. M., "Simply Implementing Non-Rational Function Gust Filters for Real Time Simulation Applications," 41st AIAA/ASME/ASCE/AHS/ASC Structures, Structural Dynamics, and Materials Conference, AIAA-2000-1488, Atlanta, GA, 2000.

${ }^{8}$ Mason, M., Pragmatic Version Control - Using Subversion, $2^{\text {nd }}$ ed., The Pragmatic Bookshelf, Raleigh, NC, 2006. 
${ }^{9}$ Chen, P. C., Moulin, B., Ritz, E., Lee, D. H., and Zhang, Z., “CFD-based Aeroservoelastic Control for Supersonic Flutter Suppression, Gust Load Alleviation, and Ride Quality Enhancement,” AIAA Structures, Structural Dynamics, and Materials Conference, AIAA-2009-2537, Palm Springs, CA, 2009.

${ }^{10}$ Moulin, B., Ritz, E., Chen, P. C., Lee, D. H., and Zhang, Z., “CFD-based Control for Flutter Suppression, Gust Load Alleviation, and Ride Quality Enhancement for the S4T Model,” AIAA Structures, Structural Dynamics, and Materials Conference, AIAA-2010-2623, Orlando, FL, 2010.

${ }^{11}$ Roughen, K. M., and Bendiksen, O. O., “Active Flutter Suppression of the Supersonic Semispan Transport (S4T) Model,” AIAA Structures, Structural Dynamics, and Materials Conference, AIAA-2010-2621, Orlando, FL, 2010.

${ }^{12}$ Mukhopadhyay, V., Newsom, J. R., and Abel, I., “A Method for Obtaining Reduced-Order Control Laws for High-Order Systems Using Optimization Techniques,” NASA-TP-1876, 1981.

${ }^{13}$ Pototzky, A. S., "Enhanced Modeling of First-Order Plant Equations of Motion for Aeroelastic and Aeroservoelastic Applications,” AIAA Atmospheric Flight Mechanics Conference, (to be published), Toronto, Ontario, Canada, 2010.

${ }^{14}$ Barrows, D. A., "Videogrammetric Model Deformation Measurement Technique for Wind Tunnel Applications," AIAA Aerospace Sciences Meeting, AIAA-2007-1163, Reno, NV, 2007. 